\title{
A New Nonlinear Multiregression Model Based on the Lower and Upper Integrals
}

\author{
Jing Chu • Zhenyuan Wang • Yong Shi • \\ Kwong-Sak Leung
}

Received: 12 December 2012 / Revised: 14 March 2013 / Accepted: 20 April 2013 /

Published online: 19 April 2014

(C) Springer-Verlag Berlin Heidelberg 2014

\begin{abstract}
A new nonlinear multiregression model based on a pair of extreme nonlinear integrals, lower and upper integrals, is established in this paper. A complete data set of predictive attributes and the relevant objective attribute is required for estimating the regression coefficients. Due to the nonadditivity of the model, a genetic algorithm combined with the pseudo gradient search is adopted to search the optimized solution in the regression problem. Applying such a nonlinear multiregression model, an interval prediction for the value of the objective attribute can be made once a new observation of predictive attributes is available.
\end{abstract}

Keywords Signed efficiency measures $\cdot$ Nonlinear integrals $\cdot$ Multiregression $\cdot$ Soft computing $\cdot$ Linear programming $\cdot$ Data mining

\footnotetext{
J. Chu

School of Management, University of Chinese Academy of Sciences, Beijing 100190, China

J. Chu · Y. Shi

Research Center on Fictitious Economy \& Data Science, Chinese Academy of Sciences, Beijing 100190, China

Z. Wang

Department of Mathematics, University of Nebraska at Omaha, Omaha, NE 68182, USA

Y. Shi $(\bowtie)$

College of Information Science and Technology, University of Nebraska at Omaha, Omaha, NE 68182, USA

e-mail: yshi@ucas.ac.cn;yshi@unomaha.edu

K.-S. Leung

Department of Computer Science and Engineering, The Chinese University of Hong Kong, Hong Kong 999077, China
} 


\section{Introduction}

The classical aggregation tool in information fusion is the weighted sum. It is a linear model. Using this linear model needs a basic assumption: there is no interaction among the contributions from predictive attributes towards the objective attribute such that the joint contribution from a group of predictive attributes is just the simple sum of contributions from each individual attribute in the group. However, in many real problems, such an interaction cannot be ignored. Fortunately, a non-classical mathematical tool, nonadditive set function, has been successfully used to describe the above-mentioned interaction in information fusion and data mining [2,5,8,11,12,14,17]. Replacing the classical weighted sun that is the classical Lebesque integral defined on a certain finite set, the aggregation tool should be a certain type of abstract integrals, which is generally nonlinear due to the nonadditivity of the involved set function. In this case, as one of the inverse problems of the information fusion [9], the multiregression should be established in terms of nonlinear integrals. Such an idea has been recently realized in some works where the Choquet integral is adopted [3,10,14,15,17,18].

The different type of nonlinear integrals corresponds to the different partition rules that describe the different coordination scheme. Generally, when a data set is available, people do not know the concrete coordination. Thus, a new question appears: why should we choose the Choquet integral in the nonlinear multiregression model, since there are various types of nonlinear integrals, such as the lower integral and the upper integral $[13,16,17]$. The lower and upper integrals form an extreme pair, the maximum and the minimum, among all nonlinear integrals $[13,17]$. That is to say, any type of nonlinear integrals is between the lower integral and the upper integral, in terms of their values. Hence, a natural idea is to use the upper integral and the lower integral to "control" the observed values of the objective attribute and, then, establish a new type of nonlinear multiregression.

To show such a new multiregression model, this paper is arranged as follows. In Sect. 2, the concept of signed efficiency measure is recalled. Section 3 gives an overview of nonlinear integrals defined on finite sets. The new nonlinear multiregression model based on the upper and the lower integral is demonstrated in Sect. 4. In Sect. 5, the algorithm for solving this regression problem is given. Two examples are presented in Sect. 6 to explain the model and to compare with the multiregression based on the Choquet integral and the classical multiregression, as well as to show the way of using such a regression model to make an interval prediction once a new observation is available. Finally, some conclusions are listed in Sect. 7.

\section{Signed Efficiency Measures}

Denote the set of all information sources by $X$ and call it the universal set. Then $(X, \mathscr{P}(X))$ is a measurable space, where $\mathscr{P}(X)$ is the power set of $X$. In almost all real problems, the universal set is finite. For example, in any database, the number of attributes is always finite. Thus, throughout this paper we assume that $X=\left\{x_{1}, x_{2}, \ldots, x_{n}\right\}$, where each $x_{i}, i=1,2, \ldots, n$, is an attribute. In a multiregression problem, $x_{1}, x_{2}, \ldots, x_{n}$ are called predictive attributes. They are usually 
numerical. There is another numerical attribute $Y$ called the objective attribute in the database as a fusion target. The observation value of $Y$ is denoted by $y$ generally. While in classification problems, $x_{1}, x_{2}, \ldots, x_{n}$ are called feature attributes and $Y$ is the classifying attribute that is categorical.

Definition 1 Any set function, $\mu: \mathscr{P}(X) \rightarrow(-\infty, \infty]$, is called a signed efficient measure if $\mu(\varnothing)=0$, where $\varnothing$ is efficient measure is called an efficient measure. Any monotone efficient measure is called a monotone measure.

The signed efficiency measure, efficiency measure, and monotone measure are also called the signed fuzzy measure, generalized fuzzy measure, and fuzzy measure respectively $[2,7,8,11,12,17]$.

Example 1 Let $X=\left\{x_{1}, x_{2}, x_{3}\right\}$. Set function $\mu: \mathscr{P}(X) \rightarrow(-\infty, \infty]$ is given as

$$
\mu(E)= \begin{cases}0 & \text { if } E=\varnothing \\ 4 & \text { if } E=\left\{x_{1}\right\} \\ -3 & \text { if } E=\left\{x_{2}\right\} \\ -2 & \text { if } E=\left\{x_{1}, x_{2}\right\} \\ 3 & \text { if } E=\left\{x_{3}\right\} \\ 2 & \text { if } E=\left\{x_{1}, x_{3}\right\} \\ -1 & \text { if } E=\left\{x_{2}, x_{3}\right\} \\ 5 & \text { if } E=X .\end{cases}
$$

Then $\mu$ is a signed efficiency measure on $\mathscr{P}(X)$.

Any signed efficiency measure $\mu$ can be decomposed as the difference of two efficiency measures, $\mu=\mu^{+}-\mu^{-}$.

Definition 2 Let $\mu: \mathscr{P}(X) \rightarrow(-\infty, \infty$ ] be a signed efficiency measure. $\mu=$ $\mu^{+}-\mu^{-}$is called the reduced decomposition of $\mu$ if both $\mu^{+}$and $\mu^{-}$are efficiency measures on $\mathscr{P}(X)$ and $\mu^{+}(E) \cdot \mu^{-}(E)=0$ for every $E \subseteq X$.

The pair of $\mu^{+}$and $\mu^{-}$is also simply called the reduced decomposition of $\mu$, where $\mu^{+}$is called the positive part of $\mu$, while $\mu^{-}$is called the negative part of $\mu$. For any given signed efficiency measure, the reduced decomposition is unique. Equality $\mu^{+}(E) \cdot \mu^{-}(E)=0$ means that at least one of $\mu^{+}(E)$ and $\mu^{-}(E)$ must be zero. In fact,

$$
\mu^{+}(E)= \begin{cases}\mu(E) & \text { if } \mu(E) \geq 0 \\ 0 & \text { otherwise }\end{cases}
$$

and

$$
\mu^{-}(E)= \begin{cases}-\mu(E) & \text { if } \mu(E)<0 \\ 0 & \text { otherwise. }\end{cases}
$$

The reduced decomposition, $\mu^{+}$and $\mu^{-}$, of a signed efficiency measure $\mu$ is its minimal decomposition in the following sense: 
(1) $\mu^{+}$and $\mu^{-}$is a decomposition of $\mu$;

(2) if $v^{+}$and $v^{-}$is a decomposition of $\mu$, then $\mu^{+} \leq v^{+}$and $\mu^{-} \leq v^{-}$.

Example 2 Consider signed efficiency measure shown in Example 1, the reduced decomposition of $\mu$ is

$$
\mu^{+}(E)= \begin{cases}0 & \text { if } E=\varnothing \\ 4 & \text { if } E=\left\{x_{1}\right\} \\ 0 & \text { if } E=\left\{x_{2}\right\} \\ 0 & \text { if } E=\left\{x_{1}, x_{2}\right\} \\ 3 & \text { if } E=\left\{x_{3}\right\} \\ 2 & \text { if } E=\left\{x_{1}, x_{3}\right\} \\ 0 & \text { if } E=\left\{x_{2}, x_{3}\right\} \\ 5 & \text { if } E=X .\end{cases}
$$

and

$$
\mu^{-}(E)= \begin{cases}0 & \text { if } E=\varnothing \\ 0 & \text { if } E=\left\{x_{1}\right\} \\ 3 & \text { if } E=\left\{x_{2}\right\} \\ 2 & \text { if } E=\left\{x_{1}, x_{2}\right\} \\ 0 & \text { if } E=\left\{x_{3}\right\} \\ 0 & \text { if } E=\left\{x_{1}, x_{3}\right\} \\ 1 & \text { if } E=\left\{x_{2}, x_{3}\right\} \\ 0 & \text { if } E=X .\end{cases}
$$

The following example explains that a signed efficiency measure can be used to describe the interaction among the contributions from the information sources towards a certain target. A similar example of workers appeared in [7] first.

Example 3 Let $X=\left\{x_{1}, x_{2}, x_{3}\right\}$ be the set of three workers. They are hired for manufacturing a certain type of toys. Their individual and joint efficiency $\mu: \mathscr{P}(X) \rightarrow$ $[0, \infty)$ is given as follows:

$$
\mu(E)= \begin{cases}0 & \text { if } E=\varnothing \\ 5 & \text { if } E=\left\{x_{1}\right\} \\ 3 & \text { if } E=\left\{x_{2}\right\} \\ 10 & \text { if } E=\left\{x_{1}, x_{2}\right\} \\ 4 & \text { if } E=\left\{x_{3}\right\} \\ 4 & \text { if } E=\left\{x_{1}, x_{3}\right\} \\ 6 & \text { if } E=\left\{x_{2}, x_{3}\right\} \\ 9 & \text { if } E=X .\end{cases}
$$

Set function $\mu$ is an efficiency measure that describes the interaction among the contributions from individual workers towards the target, the total number of toys manufactured by these workers. In this example, $\mu\left(\left\{x_{1}, x_{2}\right\}\right)>\mu\left(\left\{x_{1}\right\}\right)+\mu\left(\left\{x_{2}\right\}\right)$ shows that workers $x_{1}$ and $x_{2}$ cooperate well and, therefore, the interaction between 
their contributions is mutually promoting. While $\mu\left(\left\{x_{1}, x_{3}\right\}\right)<\mu\left(\left\{x_{1}\right\}\right)+\mu\left(\left\{x_{3}\right\}\right)$, even $\mu\left(\left\{x_{1}, x_{3}\right\}\right)<\mu\left(\left\{x_{1}\right\}\right)$, shows that workers $x_{1}$ and $x_{3}$ cooperate very bad and the interaction between their contributions is mutually inhibitive. This set function is not monotonic.

\section{Nonlinear Integrals on Finite Sets}

Let $X=\left\{x_{1}, x_{2}, \ldots, x_{n}\right\}, \mu: \mathscr{P}(X) \rightarrow(-\infty, \infty)$ and $v: \mathscr{P}(X) \rightarrow(-\infty, \infty)$ be signed efficiency measures, and $f: X \rightarrow[0, \infty)$ and $g: X \rightarrow[0, \infty)$ be nonnegative functions.

Definition 3 ([13]) A set function $\pi: \mathscr{P}(X)-\{\varnothing\} \rightarrow[0, \infty)$ is called a partition of $f$ if

$$
f(x)=\sum_{A \mid x \in A \subseteq X} \pi(A) \quad \forall x \in X .
$$

Definition 4 ([13]) Each type of integrals with respect to $\mu$ is characterized by a rule $r$, by which, for any given $f$, a partition $\pi$ of $f$ can be obtained. Regarding both $\pi$ and $\mu$ as $\left(2^{n}-1\right)$-dimensional vectors, the value of the integral of $f$ under rule $r$, denoted by (r) $\int f d \mu$ and called the $r$-integral of $f$ with respect to $\mu$, is the inner product of vectors $\pi$ and $\mu$, that is, (r) $\int f d \mu=\pi \cdot \mu$, where (r) indicates the type of integral.

The $r$-integrals have the following properties that the classical Lebesgue integral has.

(R1) (r) $\int c f d \mu=c \cdot(\mathrm{r}) \int f d \mu \quad \forall c \in[0, \infty)$,

(R2) (r) $\int f d \mu \leq$ (r) $\int g d \mu$ if $\mu \geq 0$ and $f \leq g$,

(R3) (r) $\int f d \mu \leq$ (r) $\int f d v$ if $0 \leq \mu \leq v$.

Properties (R2) and (R3) are called the monotonicity of the $r$-integral.

However, the $r$-integrals given in Definition 4 are usually nonlinear with respect to the integrand, that is, equality

$$
\text { (r) } \int(f+g) d \mu=(r) \int f d \mu+(r) \int g d \mu
$$

may not hold. A counterexample can be found in [13].

Among infinitely many types of $r$-integrals, the Lebesgue-like integral (simply called the Lebesgue integral [4], if there is no confusion) and the Choquet integral $[1,2,7,10-12,17]$ is a pair of extreme nonlinear integrals in terms of the manner of coordination among attributes [13]. The upper and the lower integrals given in Definition 5 is another pair of extreme nonlinear integrals in terms of the amount of integration value. 
Definition 5 ([12,13,17]) The upper integral of $f$ with respect to $\mu$, denoted by (U) $\int f d \mu$, is defined by

$$
\text { (U) } \int f d \mu=\sup \left\{\sum_{j=1}^{2^{n}-1} a_{j} \cdot \mu\left(A_{j}\right) \mid \sum_{j=1}^{2^{n}-1} a_{j} \chi_{A_{j}}=f\right\} \text {, }
$$

while the lower integral of $f$ with respect to $\mu$, denoted by (L) $\int f d \mu$, is defined by

$$
\text { (L) } \int f d \mu=\inf \left\{\sum_{j=1}^{2^{n}-1} a_{j} \cdot \mu\left(A_{j}\right) \mid \sum_{j=1}^{2^{n}-1} a_{j} \chi_{A_{j}}=f\right\} \text {, }
$$

where $a_{j} \geq 0$ and $A_{j}=\bigcup_{i: j_{i}=1}\left\{x_{i}\right\}$ if $j$ is expressed in binary digits as $j_{n} j_{n-1} \cdots j_{1}$ for every $j=1,2, \ldots, 2^{n}-1$.

The calculation of the upper and the lower integrals is just the procedure of solving the following linear programming problems respectively:

$$
\begin{aligned}
\text { Maximize (or Minimize) } & z=\sum_{j=1}^{2^{n}-1} a_{j} \cdot \mu_{j} \\
\text { subject to } & \sum_{j=1}^{2^{n}-1} a_{j} \chi_{A_{j}}\left(x_{i}\right)=f\left(x_{i}\right), \quad i=1,2, \cdots, n \\
& a_{j} \geq 0, \quad j=1,2, \cdots, 2^{n}-1,
\end{aligned}
$$

where $\mu_{j}=\mu\left(A_{j}\right)$ for $j=1,2, \ldots, 2^{n}-1$, and $a_{1}, a_{2}, \ldots, a_{2_{n}-1}$ are unknown parameters. The above $n$ constraints can also be rewritten as

$$
\sum_{j: x \in A_{j} \subseteq X} a_{j}=f(x) \quad \forall x \in X
$$

Defining set function $\pi: \mathscr{P}(X) \rightarrow[0, \infty)$ by $\pi\left(A_{j}\right)=\pi_{j}=a_{j}$ for $j=$ $1,2, \ldots, 2^{n}-1$, we may see that $\pi$ is a partition of $f$. So, the upper and the lower integrals are just two special types of r-integral. Its corresponding partitioning rules are "divide the integrand in such a way that the integration value is maximized" and "divide the integrand in such a way that the integration value is minimized" respectively.

From the definition above, we may have a property of the upper and the lower integrals directly.

Property (UL1) For any $r$-integral, (L) $\int f d \mu \leq$ (r) $\int f d \mu \leq$ (U) $\int f d \mu$. 
Example 4 We use the data given in Example 3. Assume that these workers are hired for 3,8 , and 5 hours respectively, that is,

$$
f(x)= \begin{cases}3 & \text { if } x=x_{1} \\ 8 & \text { if } x=x_{2} \\ 5 & \text { if } x=x_{3}\end{cases}
$$

in a certain day. If there is an excellent manger who can well arrange their work, then the number of manufactured toys in this day may be, from formula (1), as many as

$$
\text { (U) } \int f d \mu=3 \times \mu\left(\left\{x_{1}, x_{2}\right\}\right)+5 \times \mu\left(\left\{x_{2}\right\}\right)+5 \times \mu\left(\left\{x_{3}\right\}\right)=65 \text {. }
$$

Anyway, these three workers can manufacture at least

$$
\text { (L) } \int f d \mu=3 \times \mu\left(\left\{x_{1}, x_{3}\right\}\right)+2 \times \mu\left(\left\{x_{2}, x_{3}\right\}\right)+6 \times \mu\left(\left\{x_{2}\right\}\right)=42
$$

toys. As for the other two types of nonlinear integrals, the Lebesgue integral and the Choquet integral, of function $f$, we have

$$
\int f d \mu=3 \times \mu\left(\left\{x_{1}\right\}\right)+8 \times \mu\left(\left\{x_{2}\right\}\right)+5 \times \mu\left(\left\{x_{3}\right\}\right)=54
$$

and

$$
\text { (C) } \int f d \mu=3 \times \mu\left(\left\{x_{1}, x_{2}, x_{3}\right\}\right)+2 \times \mu\left(\left\{x_{2}, x_{3}\right\}\right)+3 \times \mu\left(\left\{x_{2}\right\}\right)=48 \text {. }
$$

These results also confirm Property (UL1).

In general case, the difference between the upper integral and the lower integral, (U) $\int 1 d \mu-(\mathrm{L}) \int 1 d \mu$, can be used to indicate the uncertainty associated with signed efficiency measure $\mu$. As a special case, when $\mu$ is additive, any $r$-integral is linear with respect to the integrand, and the uncertainty associated with any additive measure is 0 . Thus, the nonlinear integrals, including the upper integral, the lower integral, and the Choquet integral, are the same as the Lebesgue integral.

\section{Nonlinear Multiregression Based on the Lower and Upper Integrals}

One of the nonlinear integrals, the Choquet integral, has been applied as the aggregation tool in multiregressions $[10,14,15,17,18]$. From Sect. 3, we have known that the Choquet integral is just one of the nonlinear integrals. It has a very special cooperation manner, maximal cooperation, among predictive attributes. Hence, a new question appears: for a given data set, why such a cooperation manner is suitable? That is, why should we adopt the Choquet integral but not some type of the nonlinear integrals else? Indeed, we have no sufficient reason to defend the choice of the Choquet integral, 
which exists in our previous works. If there is no additional information focused on the cooperation manner of the predictive attributes, we are really unable to say what the most suitable one is among all of $r$-integrals. Fortunately, any $r$-integrals are dominated by the upper integral and the lower integral. Hence, we may use them to roughly represent any actual $r$-integral and to describe the possible error.

Before establishing a model of multiregression based on the lower and upper integrals, we need to recall the concept of interval number.

Definition 6 Any closed interval $[a, b]$ is called an interval number, where $a$ and $b$ are real numbers satisfying $a \leq b$.

Any real number $a$ is a special case of interval numbers. It can be written as $[a, a]$. The arithmetical operators for interval numbers have been discussed in literature, such as [17].

To consider the regression problem, assume that a complete data set with the form

$$
\begin{array}{lllll}
x_{1} & x_{2} & \ldots & x_{n} & Y \\
\hline f_{11} & f_{12} & \ldots & f_{1 n} & y_{1} \\
f_{21} & f_{22} & \ldots & f_{2 n} & y_{2} \\
\vdots & & & & \\
f_{l 1} & f_{l 2} & \ldots & f_{l n} & y_{l}
\end{array}
$$

is available, where $Y$ is the target attribute, the $j$ th row

$$
f_{j 1} f_{j 2} \ldots f_{j n} y_{j}
$$

is the $j$ th observation of attributes $x_{1}, x_{2}, \ldots, x_{n}$ and $Y, j=1,2, \ldots, l$. Positive integer $l$ represents the size of the data, and should be much larger than $2^{n}$. Each observation of $x_{1}, x_{2}, \ldots, x_{n}$ can be regarded as a function $f: X \rightarrow(-\infty, \infty)$. Thus, the $j$-th observation of $x_{1}, x_{2}, \ldots, x_{n}$ is denoted by $f_{j}$, and we write $f_{j i}=$ $f_{j}\left(x_{i}\right), i=1,2, \ldots, n$ for $j=1,2, \ldots, l$.

Now we assume $f_{j} \geq 0, j=1,2, \ldots, l$, first. To describe how attribute $Y$ depends on attributes $x_{1}, x_{2}, \ldots, x_{n}$, a new multiregression model cam be expressed as follows:

$$
Y=c+\left[(\mathrm{L}) \int(a+b f) d \mu,(\mathrm{U}) \int(a+b f) d \mu\right]+N\left(0, \sigma^{2}\right),
$$

where $c$ is a constant, $N\left(0, \sigma^{2}\right)$ is a normally distributed random perturbation with mean 0 and variance $\sigma^{2}$, and [(L) $\int(a+b f) d \mu$, (U) $\left.\int(a+b f) d \mu\right]$ is an interval number, in which functions $a: X \rightarrow(-\infty, \infty)$ and $b: X \rightarrow(-\infty, \infty)$ can be expressed as $n$-dimensional vectors, i.e., $a=\left(a_{1}, a_{2}, \ldots, a_{n}\right)$ and $b=$ $\left(b_{1}, b_{2}, \ldots, b_{n}\right)$, and are used to balance the various phases and scales of predictive attributes, while $\mu$ is a signed efficiency measure. Functions $a$ and $b$ should satisfy the following constraints: 


$$
\begin{aligned}
& \min _{1 \leq i \leq n} a_{i}=0 \\
& b_{i} \geq 0 \text { for } i=1,2, \ldots, n, \text { with } \max _{1 \leq i \leq n} b_{i}=1 .
\end{aligned}
$$

In this regression model, constant $c$, vectors $a$ and $b$, and signed efficiency measure $\mu$ are unknown parameters and are called regression coefficients. The model is nonlinear generally. Once the data set shown in (4) with sufficient large size is available, these parameters can be optimally determined by minimizing the total squared error expressed as

$$
e^{2}=\sum_{j=1}^{l}\left(e_{1 j}^{2}+e_{2 j}^{2}\right)
$$

where

$$
e_{1 j}=\left\{\begin{array}{l}
0 \text { if } y_{j}-c \in\left[(\mathrm{L}) \int\left(a+b f_{j}\right) d \mu,(\mathrm{U}) \int\left(a+b f_{j}\right) d \mu\right] \\
\min \left(\left|y_{j}-c-(\mathrm{L}) \int\left(a+b f_{j}\right) d \mu\right|,\left|y_{j}-c-(\mathrm{U}) \int\left(a+b f_{j}\right) d \mu\right|\right) \text { otherwise }
\end{array}\right.
$$

that describes the random error, and

$$
e_{2 j}=\frac{1}{2}\left((\mathrm{U}) \int\left(a+b f_{j}\right) d \mu-(\mathrm{L}) \int\left(a+b f_{j}\right) d \mu\right)
$$

that describes the uncertainty associated with the signed efficiency measure $\mu$. The higher level the uncertainty is, the larger the interval length will be.

As a constraint by reducing the half length of the interval from the lower integral to the upper integral, the second part of the error, $\sum_{j=1}^{l} e_{2 j}^{2}$, is important. Actually, without this constraint, the optimal regression coefficients tend to an extreme case that the interval from the lower integral to the upper integral is large enough to cover the value of $y$ for every sample in the data set. This means the randomness of the observed data is not considered at all. An example stated in Sect. 5 shows it clearly. Conversely, if the whole length of the interval from the lower integral to the upper integral is adopted in $e_{2 j}$, the optimal solution of $\mu$ is additive, that is, $e_{2 j}=0$. This means that the uncertainty is zero and, therefore, the results of the regression coincide with the classical linear regression model. To avoid the optimization process automatically tend to these two undesired extreme cases, no randomness and no uncertainty, and to be convenient for comparing with the classical multiregression, we adopted the half length of the interval with boundaries of the lower and upper integrals.

After determining all regression coefficients, once a new observation $f$ is available, the prediction for the target $Y$ is an interval number

$$
\hat{Y}=\left[c+(\mathrm{L}) \int(a+b f) d \mu, c+(\mathrm{U}) \int(a+b f) d \mu\right] .
$$


Variance $\sigma^{2}$ can be estimated by

$$
\hat{\sigma}^{2}=\frac{\sum_{j=1}^{l}\left(e_{1 j}^{2}+e_{2 j}^{2}\right)}{l-2^{n}-2 n+2},
$$

where $2^{n}+2 n-2$ is the degree of freedom of the model.

In case the assumption $f_{j} \geq 0 \forall j=1,2, \ldots, l$ is not true, denoting $m(f)=$ $\min _{j=1,2, \ldots, l ; i=1,2, \ldots, n} f_{j i}$, we may replace $f_{j i}$ by $f_{j i}-m(f)$ for each $j=1,2, \ldots, l$ and $i=1,2, \ldots, n$.

\section{A Genetic Algorithm Combined with Pseudo Gradient Search Algorithm}

The optimization presented in Sect. 4 is nonlinear with respect to unknown regression coefficients. Using only an analytical and/or algebraic method to solve such an optimization problem is difficult. We should use some soft computing techniques to search for an approximate optimal solution numerically. The genetic algorithm is one of the feasible methods. However, its performance is not ideal sometimes if there are too many genes. This is just the situation appearing in the optimization problem in Sect. 4. One possible method to improve the performance of the optimization is the pseudo gradient search shown in [17]. It is a local optimization search with initial point obtained by the genetic algorithm. By this method, we can not only decrease $e^{2}$ in Sect. 4, but also make the optimal solution stable and rather close to the global maximizer / minimizer. The algorithm consists of two parts as follows:

In the first part, a genetic algorithm is adopted. Each parameter is presented by a gene that is denoted by $g$ with a subscript. Let genes $g_{1}, g_{2}, \cdots, g_{n}$ present $a_{1}, a_{2}, \cdots, a_{n}$, genes $g_{n+1}, g_{n+2}, \cdots, g_{2 n}$ present $b_{1}, b_{2}, \cdots, b_{n}$, gene $g_{2 n+1}$ presents $c$, and genes $g_{2 n+2}, g_{2 n+3}, \cdots, g_{2^{n}+2 n}$ present $\mu_{1}, \mu_{2}, \cdots, \mu_{2^{n}-1}$ respectively, where $\mu_{j}$ denotes $\mu\left(A_{j}\right)$, in which $A_{j}=\bigcup_{i: j_{i}=1}\left\{x_{i}\right\}$ if $j$ is expressed in binary digits as $j_{n} j_{n-1} \cdots j_{1}$ for every $j=1,2, \cdots, 2^{n}-1$. Genes $g_{1}, g_{2}, \cdots, g_{2^{n}+2 n}$ form a chromosome. If the binary code is adopted, noting that the actual value of each gene is in $[0,1]$, the decoding formulas should be

$$
\begin{aligned}
a_{i} & =\frac{g_{i}-m(g)}{\left(1-g_{i}\right)(1-m(g))}, \\
b_{i} & =\frac{g_{n+i}}{M(g)}, \\
c & =\tan \left[\left(g_{2 n+1}-\frac{1}{2}\right) \pi\right],
\end{aligned}
$$

and

$$
\mu_{k}=\tan \left[\left(g_{2 n+k+1}-\frac{1}{2}\right) \pi\right]
$$


for $i=1,2, \ldots, n$ and $k=1,2, \ldots, 2^{n}-1$, where $m(g)=\min _{1 \leq i \leq n} g_{i}$ and $M(g)=$ $\max _{1 \leq i \leq n} g_{n+i}$.

Once the convergence speed is too low, that means the best individual in the population doesn't change for 20 generations, we can turn to the second part, the algorithm of pseudo gradient search, to find the approximate local minimizer. Let $g^{(0)}=\left(g_{1}^{(0)}, g_{2}^{(0)}, \cdots, g_{2^{n}+2 n}^{(0)}\right)$ be the initial point, which is the best chromosome obtained in the first part. Then, determine the pseudo gradient through a learning procedure. Along this direction, a much better point can be found by another onedimensional learning procedure shown in [17]. This point is used as the starting point of the next iteration.

The algorithm of the pseudo gradient search converges fast, with 5-10 iterations or less usually.

\section{Examples}

A real data set given in a classical probability and statistic text book [6] is used to evaluate the performance of the nonlinear multiregression based on lower and upper integrals. By using the real data set, we test and verify that our model makes sense. We also give the compares among the multiregression based on lower and upper integrals and other multiregression models, such as the classical linear multiregression and the nonlinear multiregression based on the Choquet integral.

The data set is shown in Table 1. It includes 3 features 20 records, with background that an engineer at a semiconductor company wants to model the relationship between the device $\operatorname{HFE}(y)$ and three feature attributes: Emitter-RS $\left(x_{1}\right)$, Base-RS $\left(x_{2}\right)$, and Emitter-to Base-RS $\left(x_{3}\right)$. As we can hardly find a larger real-world data set with 3 feature attributes in literature, this data set is adopted without any better choice though its size is not sufficiently large.

Based on the data set shown in Table 1, running the programming involving a genetic algorithm combined with a pseudo gradient search algorithm, the value of the signed efficiency measure $\mu$, with $c, a_{i}, b_{i}$, where $i=1,2,3$, is obtained as shown in Table 2 .

The convergence of the multiregression based on lower and upper integral is shown in Fig. 1, where the population size used in the genetic algorithm is 200 Table 3.

As another multiregression model based on a nonlinear integral, the model based on the Choquet integral with averaged residual 1.855, which is $40.392 \%$ lower than classical linear multiregression model, can reduce the total error significantly. The parameters decided by the model are as below:

The residuals of these three different regression models are listed in Table 4, where $l$ is the sample size.

From Table 4, we can see that both multiregressions based on the lower and upper integrals and the Choquet integral have lower average residual than that of the classical linear multiregression. This means that the interaction among the contribution rates from predictive attributes towards the target, $y$ (HFE), is significant. The deviation described by the above-mentioned interaction is misunderstood in the classical multiregression model as a part of the residual. The advantage of the multiregression based 
Table 1 Real world data set

\begin{tabular}{llllc}
\hline$j$ & $f_{j 1}$ & $f_{j 2}$ & $f_{j 3}$ & $y$ \\
\hline 1 & 14.62 & 226 & 7 & 128.4 \\
2 & 15.63 & 220 & 3.375 & 52.62 \\
3 & 14.62 & 217.4 & 6.375 & 113.9 \\
4 & 15 & 220 & 6 & 98.01 \\
5 & 14.5 & 226.5 & 7.625 & 139.9 \\
6 & 15.25 & 224.1 & 6 & 102.6 \\
7 & 16.12 & 220.5 & 3.375 & 48.14 \\
8 & 15.13 & 223.5 & 6.125 & 109.6 \\
9 & 15.5 & 217.6 & 5 & 82.68 \\
10 & 15.13 & 228.5 & 6.625 & 112.6 \\
11 & 15.5 & 230.2 & 5.75 & 97.52 \\
12 & 16.12 & 226.5 & 3.75 & 59.06 \\
13 & 15.13 & 226.6 & 6.125 & 111.8 \\
14 & 15.63 & 225.6 & 5.375 & 89.09 \\
15 & 15.38 & 229.7 & 5.875 & 101 \\
16 & 14.38 & 234 & 8.875 & 171.9 \\
17 & 15.5 & 230 & 4 & 66.8 \\
18 & 14.25 & 224.3 & 8 & 157.1 \\
19 & 14.5 & 240.5 & 10.87 & 208.4 \\
20 & 14.62 & 223.7 & 7.375 & 133.4 \\
\hline & & & &
\end{tabular}

Table 2 The estimated values of signed efficient measure $\mu$ and other parameters

\begin{tabular}{lllll}
\hline$a_{1}=0$ & $a_{2}=1.1259$ & $a_{3}=0.0721$ & $b_{1}=0.9928$ & $b_{2}=0.9918$ \\
\hline$b_{3}=1$ & $c=45.770$ & $\mu(\varnothing)=0$ & $\mu\left(x_{1}\right)=-9.735$ & $\mu\left(x_{2}\right)=0.428$ \\
$\mu\left(x_{3}\right)=18.238$ & $\mu\left(x_{1}, x_{2}\right)=-9.440$ & $\mu\left(x_{1}, x_{3}\right)=8.509$ & $\mu\left(x_{2}, x_{3}\right)=18.780$ & $\begin{array}{c}\mu\left(x_{1}, x_{2}, x_{3}\right) \\
=8.921\end{array}$ \\
\hline
\end{tabular}

Fig. 1 The convergence of the multiregession based on lower and upper integrals

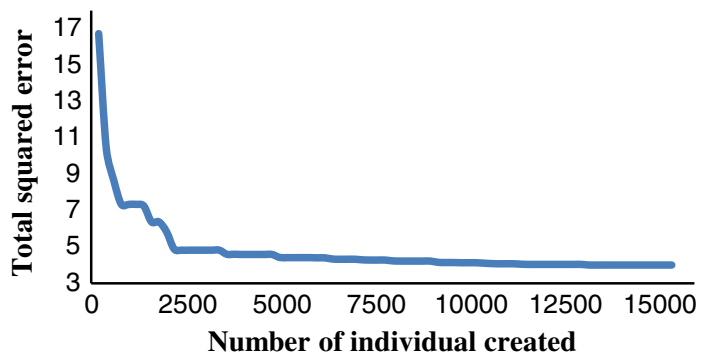

on the lower and upper integrals is more significant when the data set size is large enough, as the degree of freedom can affect the result slightly.

In some extreme cases, the Choquet integral is either the lower integral or the upper integral [17]. Thus, the main difference between the residuals of the multiregression based on the lower and upper integrals and that based on the Choquet integral is 


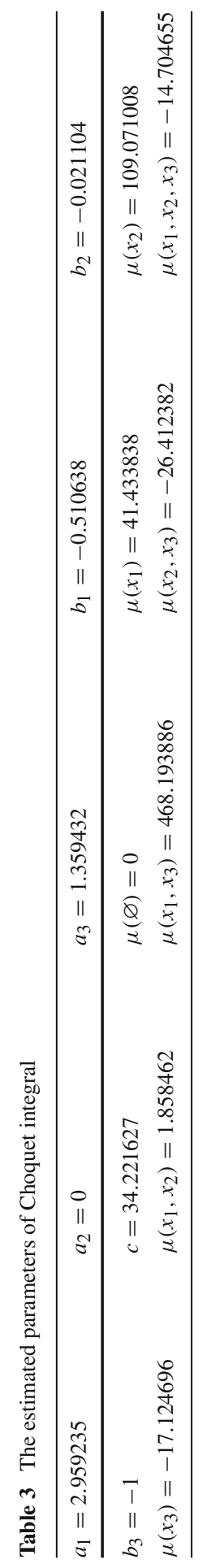


Table 4 The residuals of three different regression models

\begin{tabular}{ll}
\hline Regression type & Average residual (root-mean-square error) \\
\hline Based on the lower and upper integral & $e=\sqrt{\sum_{j=1}^{l}\left(e_{1 j}^{2}+e_{2 j}^{2}\right) / l}=2.322$, \\
Based on the Choquet integral & where $\sum_{j=1}^{l} e_{1 j}^{2}=71.207, \sum_{j=1}^{l} e_{2 j}^{2}=36.627$ \\
Classical linear multiregression & $e=\sqrt{\sum_{j=1}^{l} e_{j}^{2} / l}=1.855$, where $\sum_{j=1}^{l} e_{j}^{2}=68.796$ \\
& $e=\sqrt{\sum_{j=1}^{l} e_{j}^{2} / l}=3.112$, where $\sum_{j=1}^{l} e_{j}^{2}=193.725$ \\
\hline
\end{tabular}

Table 5 Prediction of three different regression models

\begin{tabular}{lllll}
\hline Regression type & predicted HFE $(\hat{y})$ & Emitter-RS $\left(x_{1}\right)$ & Base-RS $\left(x_{2}\right)$ & Emitter-to Base-RS $\left(x_{3}\right)$ \\
\hline $\begin{array}{c}\text { Based on the lower } \\
\text { and upper integral }\end{array}$ & {$[89.654,92.243]$} & 15.13 & 226.06 & 5.17 \\
$\begin{array}{c}\text { Based on the Choquet } \\
\text { integral }\end{array}$ & 89.898 & & \\
$\begin{array}{c}\text { Classical linear } \\
\text { multiregression }\end{array}$ & 90.990 & & \\
\hline
\end{tabular}

the half-length of the interval from the lower integral to the upper integral. Without missing the regression accuracy rate, the multiregression based on the lower and upper integrals can give more information by giving an interval prediction.

The next example shows how the multiregression model based on the lower and upper integrals works in prediction. As we get all the regression coefficients through the learning procedure based on the given data set, we can predicate the target $\mathrm{HFE}(\mathrm{y})$ by a form of interval once a new observation of the predictive attributes, Emitter-RS $\left(x_{1}\right)$, Base- $\mathrm{RS}\left(x_{2}\right)$, and Emitter-to Base- $\mathrm{RS}\left(x_{3}\right)$,is available.

For comparing the prediction difference among the multiregression model based on the lower and upper integrals, the multiregression based on the Choquet integral, and classical linear multiregression, we list all of these three predicting results based on the same data set in Table 5 .

From Table 5, we can see that the prediction of HFE is between 89.654 and 92.243 by multiregression model based on the lower and upper integrals. It means that the HFE is 89.654 in one possible extreme case and 92.243 in another possible extreme case. The predictions of the other two regression models are in this interval.

\section{Conclusions}

The multiregression model based on lower and upper integrals can not only show the contributions of feature attributes towards the target, but also considers the interaction among the contribution rates from various predictive attributes towards the target. 
Based on the given data set, through a learning procedure, we can estimate the values of the nonadditive measures, which describe the above-mentioned interaction. The difference between the lower integral and the upper integral is owed to the interaction, so the multiregression based on the lower and upper integrals can find out the deviation described by the interaction. This deviation is misunderstood as a part of residual due to the randomness in the classical linear multiregression. If the interaction is not significant, this difference is quite small. In that case, the result of the multiregression based on the lower and upper integrals is closed with that of the classical multiregression.

In the example shown in Sect. 6, we adopt an algorithm consisting of a genetic algorithm combined with a pseudo gradient search, which overcomes premature phenomenon that usually appears in the genetic algorithm and is regarded as its essential weakness.

The multiregression based on the lower and upper integrals is a new method for data analysis. As it gives an predictive interval without missing the accuracy, this method is powerful, especially to data sets for which the classical linear multiregression fails by leaving a large residual.

Acknowledgments This research is partially supported by National Science Foundation of China \#70921061 and \#71110107026, and by the CAS/SAFEA International Partnership Program for Creative Research Teams.

\section{References}

1. Choquet G (1954) Theory of capacities. Annales de l'Institut Fourier 5:131-295

2. Denneberg D (1994) Non-additive measure and integral. Kluwer Academic, Dordrecht

3. Grabisch M, Kojadinovic I, Meyer P (2008) A review of methods for capacity identification in Choquet integral based multi-attribute utility theory applications of the Kappalab R package. Eur J Oper Res 186:766-785

4. Halmos PR (1967) Measure Theory. Van Nostrand, New York

5. Lee S, Liu H, Chen S, Jheng Y (2011) Importance-performance analysis based on Choquet integral with respect to L-measure. Appl Mech Mater 44-47:3844-3848

6. Montgomery DC, Runger GC (1998) Applied statistics and probability for engineers, 2nd edn. Wiley, New York

7. Murofushi T, Sugeno M, Machida M (1994) Non-monotonic fuzzy measures and the Choquet integral. Fuzzy Sets Syst 64:73-86

8. Sugeno M (1974) Theory of fuzzy integral and its applications. Ph.D. dissertation, Tokyo Institute of Technology

9. Tzeng G, Yang Y, Lin C, Chen C (2005) Hierarchical MADM with fuzzy integral for evaluating enterprise intranet web sites. Inf Sci 169:409-426

10. Wang Z (2003) A new genetic algorithm for nonlinear multiregressions based on generalized Choquet integrals. In: Proceedings of FUZZ-IEEE, pp 819-821

11. Wang Z, Klir GJ (1992) Fuzzy measure theory. Plenum, New York

12. Wang Z, Klir GJ (2008) Generalized measure theory. Springer, New York

13. Wang Z, Leung KS, Klir GJ (2006) Integration on finite sets. Int J Intell Syst 21:1073-1092

14. Wang Z, Leung KS, Klir GJ (2005) Applying fuzzy measures and nonlinear integrals in data mining. Fuzzy Sets Syst 156:371-380

15. Wang Z, Leung KS, Wong ML, Fang J, Xu K (2000) Nonlinear nonnegative multiregressions based on Choquet integrals. Int J Approx Reason 25:71-87

16. Wang Z, Li W, Lee KH, Leung KS (2008) Lower integrals and upper integrals with respect to nonadditive set functions. Fuzzy Sets Syst 159:646-660

17. Wang Z, Yang R, Leung KS (2010) Nonlinear integrals and their applications in data mining. World Scientific, Singapore 
18. Yang R, Wang Z, Heng PA, Leung KS (2008) Fuzzified Choquet integral with fuzzy-valued integrand and its application on temperature prediction. IEEE T. SMCB 38(2):367-380

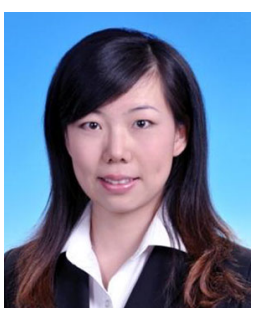

Jing Chu received an MS in Management Science and Engineering from the Chinese Academy of Sciences, 2012. She currently serves at Chinese Security Company as a Chinese bond trader. She focuses on data mining, fuzzy measures and fuzzy integrals and optimization and business intelligence. She has been a visiting scholar in University of Nebraska at Omaha, USA in 2011.

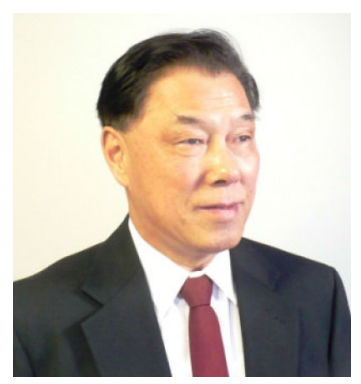

Zhenyuan Wang graduated from Fudan University in 1962. He received his Ph.D. from the Department of Systems Science, State University of New York at Binghamton in 1991. He taught various mathematical courses in Hebei University for many years since 1962, supervised graduate students since 1978, and served as the Chair of the Mathematics Department there from 1985 to 1990. He was a visiting scholar, visiting professor, or research fellow in University Paris VI, Binghamton University (SUNY), Chinese University of Hong Kong, New Mexico State University, and University of Texas at El Paso during the period from 1979 to 2008. Currently, he is a tenured full professor in the Department of Mathematics, University of Nebraska at Omaha. He received a number of honors and awards including the title of "National expert" from the Chinese National Scientific and Technological Commission in 1986 and the "Citation Classic Award" from the Institute for Scientific Information (USA) in 2000. His research interests are nonadditive measures, nonlinear integrals, probability and statistics, optimization, soft computing, and data mining. He is the author or a co-author of more than 150 research papers and three monographs: "Fuzzy Measure Theory" (1992), "Generalized Measure Theory" (2008), and "Nonlinear Integrals and Their Applications in Data Mining" (2010).

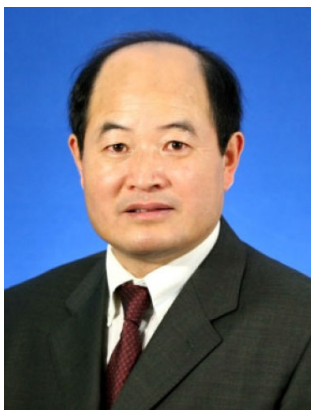

Yong Shi Senior Member of IEEE, serves as the Executive Deputy Director, Chinese Academy of Sciences Research Center on Fictitious Economy and Data Science. He has been the Union Pacific Chair of Information Technology, College of Information Science and Technology, Peter Kiewit Institute, University of Nebraska, USA. Dr. Shi ‘s research interests include business intelligence, data mining, and multiple criteria decision making. He has published more than 20 books, over 200 papers in various journals and numerous conferences/proceedings papers. He is the Editorin-Chief of International Journal of Information Technology and Decision Making (SCI), and a member of Editorial Board for a number of academic journals. Dr. Shi has received many distinguished awards including the Georg Cantor Award of the International Society on Multiple Criteria Decision Making (MCDM), 2009; Fudan Prize of Distinguished Contribution in Management, Fudan Premium Fund of Management, China, 2009; Outstanding Young Scientist Award, National Natural Science Foundation of China, 2001; and Speaker of Distinguished Visitors Program (DVP) for 1997-2000, IEEE Computer Society. He has consulted or worked on business projects for a number of international companies in data mining and knowledge management. 


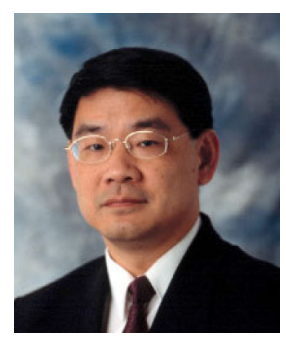

Kwong-Sak Leung received his B.Sc (Eng.) and Ph.D. degrees in 1977 and 1980, respectively, from the University of London, Queen Mary College. He worked as a senior engineer on contract R\&D at ERA Technology and later joined the Central Electricity Generating Board to work on nuclear power station simulators in England. He joined the Computer Science and Engineering Department at the Chinese University of Hong Kong in 1985, where he is currently Professor of Computer Science \& Engineering. Leung's research interests are in bioinformatics and soft computing including evolutionary computation, parallel computation, probabilistic search, information fusion and data mining, fuzzy data and knowledge engineering. He has authored and coauthored over 350 papers and 4 books in fuzzy logic and evolutionary computation. He has been chair and member of many program and organizing committees of international conferences. He is in the Editorial Board of Fuzzy Sets and Systems and an associate editor of International Journal of Intelligent Automation and Soft Computing. He is a senior member of the IEEE, a chartered engineer, a member of IET and ACM, a fellow of HKIE and a distinguished fellow of HKCS in Hong Kong. 